\title{
Digital Implementation Method of Base-band Signal of FSK Quadrature Modulation
}

\author{
Jinyu Bao \\ School of Aeronautics and Astronautics \\ UESTC \\ Chengdu, China, 611731 \\ doubaoyyxa@126.com \\ Bo Tang \\ School of Aeronautics and Astronautics \\ UESTC \\ Chengdu, China, 611731 \\ tangbocd@126.com
}

\author{
Kaiyu Qin \\ School of Aeronautics and Astronautics \\ UESTC \\ Chengdu, China,611731 \\ kyqin@uestc.edu.cn \\ Honglin Dou \\ School of Electronic Engineering \\ UESTC \\ Chengdu, China,611731 \\ hldou1021@gmail.com
}

\begin{abstract}
FSK modulation is widely used in communication system. In this paper, based on the basic formula of the communication theory, we concluded a new digital implementation method of base-band signal of 2FSK quadrature modulation formula, and realized in base-band signal simulation software based on $\mathrm{VC}++$, with which we can analyse the signal with constellation, vector and frequency spectrogram designed on our own. This new method makes it easy to build the unification system platform.
\end{abstract}

Keywords-FSK; MSK; VC++; quadrature; modulation

\section{INTRODUCTION}

Usually engineers adopt the mode of DDS to realize FSK modulation , but this kind of method can't achieve the baseband signal before FSK processing of carrier frequency modulation ,however the other modes of modulation such as QAM, PSK can easily get their base-band signal. In order to analyze the characteristics of base-band signal, we also need to get the base-band signal of FSK modulation. In the symbol mapping, we can set amplitude imbalance ratio and phase imbalance ratio of IQ according to the constellation diagram ,producing effect about the constellation diagram such as rotation, compression, distortion , to test the capacity of equipment demodulation and signal recovery.

\section{QUADRATURE MODULATION OF 2FSK}

\section{A. The Theory of Communication}

Wireless communication transfers data information through modulation to the carrier of amplitude, frequency, and phase as communication principle shows. Suppose a carrier modulated as following:

$$
S(t)=A(t) \cos \left[2 \pi f_{c} t+\theta(t)\right]
$$

$f_{c}$ is the angular frequency of carrier, $A(t)$ is the amplitude modulation information of signal, $\theta(t)$ is the phase modulation information of signal. Through the simplified deformation of triangle function, 错误! 未找到引用源。can be written also as following:

$$
S(t)=I(t) \cos \left(2 \pi f_{c} t\right)-Q(t) \sin \left(2 \pi f_{c} t\right)
$$

$I(t)$ and $Q(t)$ are as following:

$$
\begin{aligned}
& I(t)=A(t) \cos \theta(t) \\
& Q(t)=A(t) \sin \theta(t)
\end{aligned}
$$

If it is FSK modulation, frequency information represents the change of signal, the amplitude function in formula can be $A(t)=1$.

We can suppose $\theta(t)=0$, so the expression of signal shows as following:

$$
S(t)=\cos \left(2 \pi\left(f_{c}+f_{n}\right) t\right)
$$

That is,

$$
S(t)=\cos \left(2 \pi f_{c} t\right) \cos \left(2 \pi f_{n} t\right)-\sin \left(2 \pi f_{c} t\right) \sin \left(2 \pi f_{n} t\right)
$$




$$
\begin{gathered}
I(t)=\cos \left(2 \pi f_{n} t\right) \\
Q(t)=-\sin \left(2 \pi f_{n} t\right)
\end{gathered}
$$

Actually we can get $I^{2}(t)+Q^{2}(t)=1$ from $I(t)$ and $Q(t)$, that constellation chart and vector diagram are both circle through the 2FSK modulation expression.

A modulation which can produce constant envelope and continuous signal is called minimum frequency shift keying, 2FSK which has the minimum frequency difference of orthogonal signal. The phase keep continuous in neighboring symbols junction. noting for MSK.MSK is a kind of special circumstance of 2FSK which has the minimum frequency difference of orthogonal signal. The phase keeps continuous in neighboring symbols junction.

$$
\begin{gathered}
S_{C P F S K}(t)=A \cos \left(\omega_{c} t+\Delta \omega t+\theta\right) \\
\omega_{c}=\frac{\omega_{0}+\omega_{1}}{2} \\
\Delta \omega=\frac{\omega_{0}-\omega_{1}}{2}
\end{gathered}
$$

We consider that the FSK signal is orthogonal when $\mathrm{n}$ is an integral number. $\Delta \omega$ must be small in order to improve the utilization rate of frequency band. $\Delta \omega$ reaches the minimum when $n=1$. The frequency difference is half of the symbol rate, reaching the minimum of orthogonal signal. The particular choice of CPFSK is called minimum frequency shift keying(MSK).

\section{B. Discrete Expression of I and $Q$}

As the system clock $f_{\text {sys }}$ is sure, the relation between frequency required and stepping angle is as following:

$$
\begin{aligned}
& f=f_{s y s} \frac{\theta}{2 \pi} \\
& f=f_{c}+f_{n}
\end{aligned}
$$

$f$ is the frequency after modulation, $f_{c}$ is carrier frequency, $f_{n}$ is frequency shift.

$$
\theta=\theta_{c}+\theta_{n}
$$

Stepping angle is contains two parts, one is caused by carrier frequency $\theta_{\mathrm{c}}$ and the other part is caused by frequency shift is $\theta n$.

$$
\begin{aligned}
& \theta_{c}=\frac{2 \pi f_{c}}{f_{s y s}} \\
& \theta n=\frac{2 \pi f_{n}}{f_{s y s}}
\end{aligned}
$$

The system clock is larger than the rate for code or equal, meeting the following relation:

$$
f_{\text {sys }}=\text { Oversampling } * f_{\text {sym }}
$$

Oversampling is oversampling ratio.

It supposed not to be interpolate before the constellation chart deserializing.

$$
f_{\text {sys }}=f_{\text {sym }}
$$

So the frequency shift $f_{0}$ of baseband signal which we choose corresponds the stepped angle that is

$$
\theta_{0}=\frac{2 \pi f_{0}}{f_{\text {sym }}}
$$

So the frequency shift $f_{1}$ of baseband signal which we choose corresponds the stepped angle that is

$$
\theta_{1}=\frac{2 \pi f_{1}}{f_{s y m}}
$$

Applied in projects we need to achieve the discrete of $I(t) 、 Q(t)$ channel from(7)and(8)

$$
\begin{gathered}
I(t)=\cos \left(2 \pi f_{n} t\right)=\cos \left(N \theta_{n}\right) \\
Q(t)=-\sin \left(2 \pi f_{n} t\right)=-\sin \left(N \theta_{n}\right)
\end{gathered}
$$

$N=\frac{2 \pi}{\theta_{n}}, N$ must be an integer. 


\section{REALIZATION OF BASE-BAND Signal SimUlation SOFTWARE AND 2FSK}

A. The Flow Chart of the Whole Base-band Signal Simulation Software

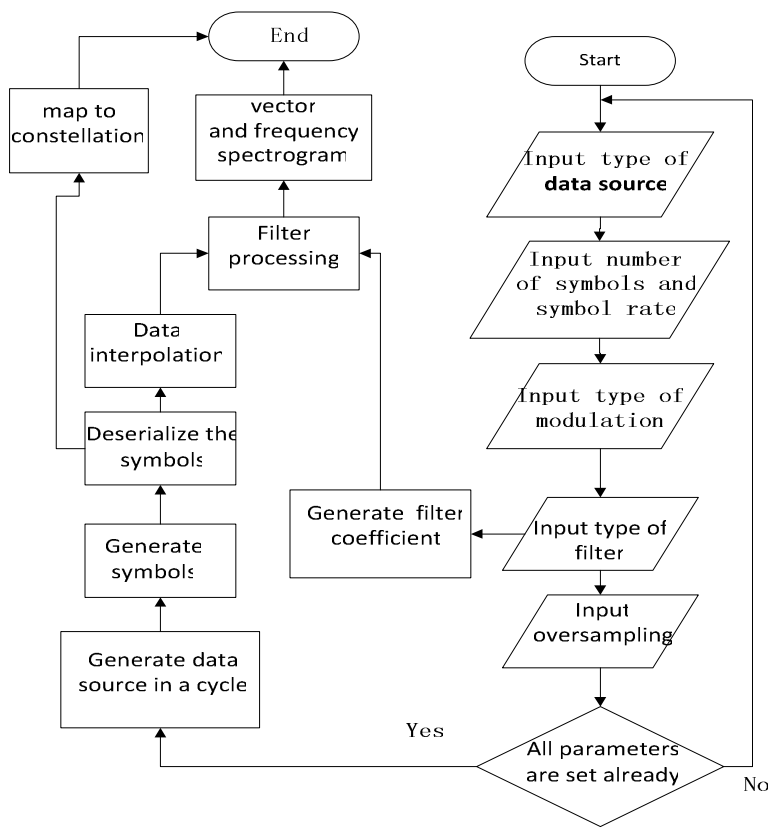

Figure 1. The flow chart of base-band signal simulation software.

B. The Implement of $2 \mathrm{FSK}$

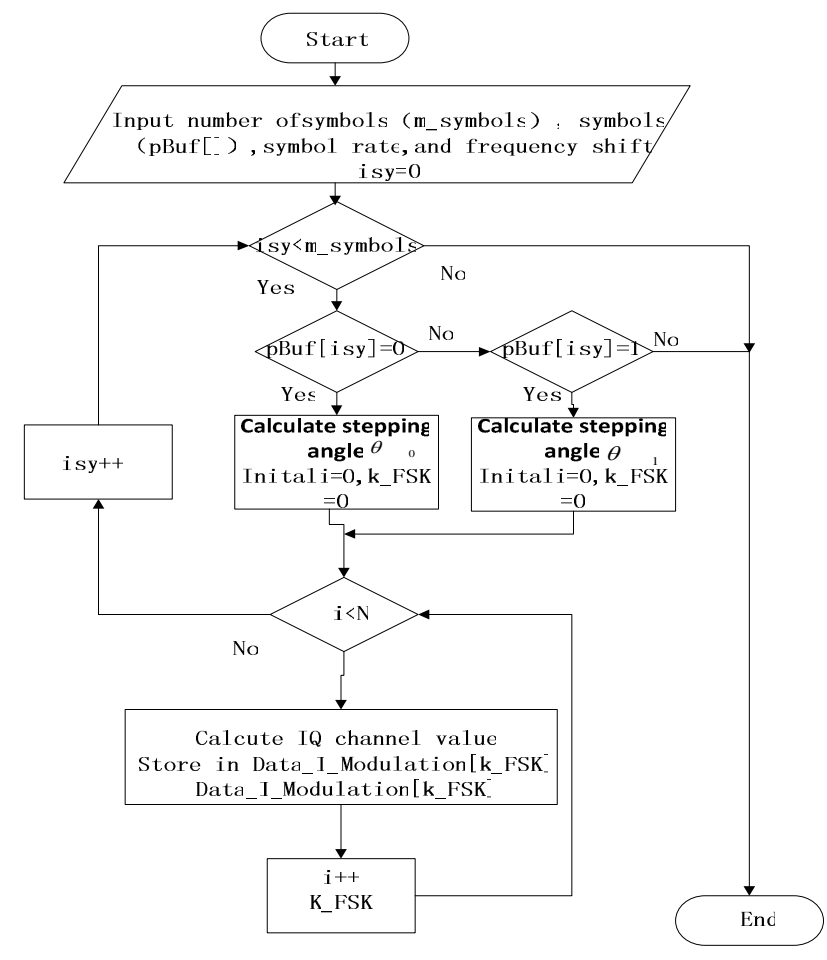

Figure 2. The flow chart of FSK implement.

\section{The Simulation Result}

Code type, code number, code rate, filter parameters, filter types, frequency shift $f_{0}$ and frequency shift $f_{1}$ are all set by users through base-band signal simulation software by themselves as follows, $f_{0}$ is $250 \mathrm{~Hz}, f_{1}$ is $-2500 \mathrm{~Hz}$, the rolloff of filter is 0.3 , length of filter coefficient is 99 .

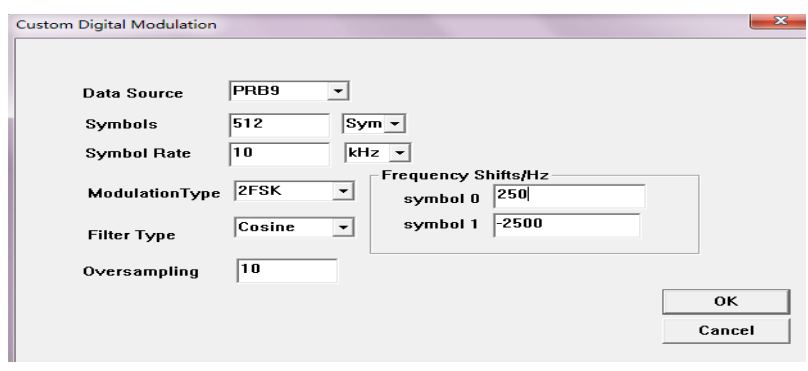

Figure 3. Parameter setting.

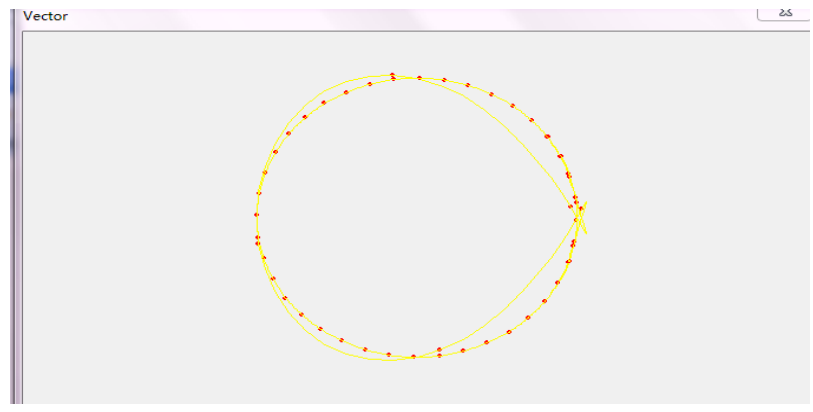

Figure 4. Vector diagram of 2FSK.

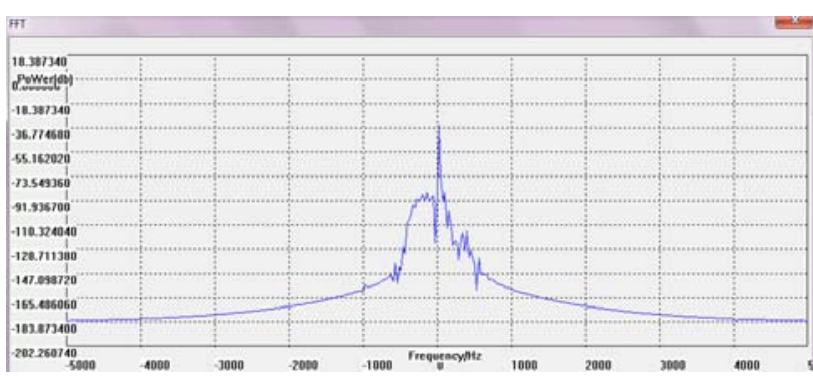

Figure 5. Frequency spectrogram of 2FSK.

In order to produce bright contrast, the modulation mode is changed to MSK. The frequency offset $f_{0}, f_{1}$ become the default value while code rate is fixed by $10 \mathrm{kHz}$,so $f_{0}$ is $2500 \mathrm{~Hz}, f_{1}$ is $-2500 \mathrm{~Hz}$, and keep the other parameters not change. 


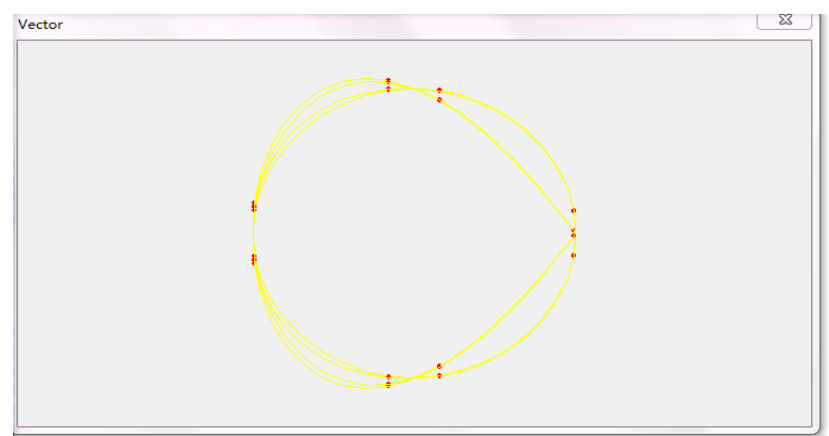

Figure 6. Vector diagram of MSK.

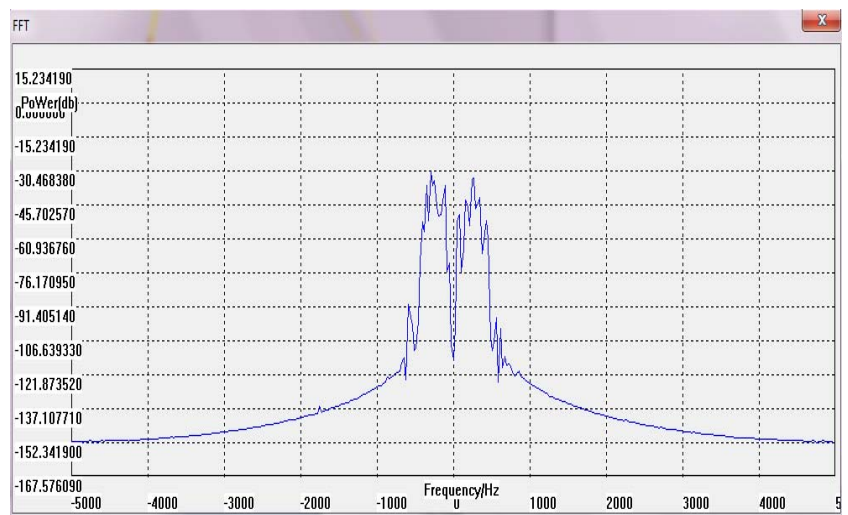

Figure 7. Frequency spectrogram of MSK.

According to this method we can easily get the base-band signal of FSK modulation, we choose different types of data source, filters, oversampling, and so on. We just need one platform even apply in hardware.

\section{CONCLUSION}

I, Q channel simulation of FSK base-band signal is realized through software on PC, what's more, the analysis of constellation chart, vector diagram and frequency spectrogram is similar with the theoretical result, which achieves the expected goal. This new method not only is good for communication system unify with other modulations such as QAM and PSK but also has the high engineering application value.

\section{REFERENCES}

[1] Shang, J, "A new software demodulation method for MSK signal based on phase information," Systems and Control in Aerospace and Astronautics, 2006. ISSCAA 2006. 1st International Symposium on, 3 pp. - 830, 19-21 Jan. 2006

[2] Gronemeyer, S and McBride, A, "MSK and Offset QPSK Modulation," Communications, IEEE Transactions on , vol.24, no.8, pp. 809- 820, Aug 1976

[3] Prabhu, V.K., "MSK and Offset QPSK Modulation with Bandlimiting Filters", Aerospace and Electronic Systems, IEEE Transactions on, On page(s): 2 - 8 Volume: AES-17, Issue: 1, Jan. 1981

[4] Simon, M., "An MSK Approach to Offset QASK," Communications, IEEE Transactions on , vol.24, no.8, pp. 921- 923, Aug 1976

[5] Lee, R., Chiu, M.and Lin, J, Communications Engineering:Essentials for Computer Scientists and Electrical Engineers , Wiley-IEEE Press, 2007. 